\title{
Spectral reflectance characterization of road environment to optimize the choice of autonomous vehicle sensors*
}

\author{
Michèle Colomb $^{1}$, Pierre Duthon ${ }^{1}$ and Frédéric Bernardin ${ }^{1}$
}

\begin{abstract}
A wide range of sensors are developed for autonomous vehicles in order to ensure safe driving conditions by detecting any relevant objects in the road environment. The sensors, cameras, Lidars and radars, cover a wide range of wavelength, ranging from visible, Near InfraRed, Short Wave InfraRed and beyond for high frequency radars. For Lidars technology, a single wavelength scans the environment. To get the most efficient detection, the choice of the wavelength must be optimized. Knowledge of the spectral reflectance of road objects at the chosen wavelength is a key point. An original application of reflectance measurement technics is proposed to characterize road objects in the range of wavelength between 350 to $2450 \mathrm{~nm}$. This investigation could lead to the definition of reference tests including a physical characterization of the environment.
\end{abstract}

\section{INTRODUCTION}

Intelligent transportation systems are facing an increase of sensors development to cope with the challenge of driving automation. The future intelligent systems of the autonomous vehicles must provide two major functions: navigation and obstacle detection in all possible use cases, ordinary but also in the most critical environment. The challenge is to provide these functions with an increased efficiency compared to the current situation where humans are at the controls of vehicles. Indeed, there is a wide technological offer that is proposed as cameras, Lidars or radars, in order to take the relevant visual informations to ensure the driving task instead of the driver. Until now, the driver has been monitoring the environment and taking the relevant information for the driving task in the visible range. With the development of autonomous driving, for automation level 3 to 5 [1] the system will have to monitor the environment, and then to guarantee the driving safety [2].

Cameras are operating in the visible range, Near InfraRed, NIR, Short Wave InfraRed, SWIR or Long Wave InfraRed, LWIR. Relevant informations are taken from the image of the road scene, that is obtained by the reflection or emission of all objects in the considered wavelengths. It is then

\footnotetext{
*The research leading to these results has received funding from the European Union under the H2020 ECSEL Program as part of the DENSE project, contract number 692449. The French government research program "Investissements d'Avenir" also contributed through the IMobS3 Laboratory of Excellence (ANR-10-LABX-16-01).

${ }^{1}$ Michèle Colomb is with Cerema, Equipe-projet STI, 8-10, rue, Bernard Palissy, F-63017, Clermont-Ferrand Cedex 2, France michele. colombecerema. fr

${ }^{2}$ Pierre Duthon is with Cerema, Equipe-projet STI, 8-10, rue, Bernard Palissy, F-63017, Clermont-Ferrand Cedex 2, France pierre.duthon@cerema.fr

${ }^{3}$ Frédéric Bernardin is with Cerema, Equipe-projet STI, 8-10, rue, Bernard Palissy, F-63017, Clermont-Ferrand Cedex 2, France fredredic.bernardinecerema. fr
}

required to investigate what is the effect of the range of wavelength on the detection of road signaling equipment as well as on the detection of relevant fixed obstacles or of vulnerable road users. The very auguring Lidars technology is using a single wavelength to scan the environment. What is the best wavelength to use to get the most efficient sensor? Available sensors are operating at $905 \mathrm{~nm}$, some studies discuss the potential benefits of using new systems operating at $1550 \mathrm{~nm}$ when used in foggy conditions [3]. When combining technologies, it is also useful to know the reflectance properties of all the objects in each considered wavelength. As a single sensor is not be able to detect all objects in all conditions, fusion methods for Lidars-camera setups are proposed to optimize the object detection by computer vision [4].

Multispectral characterization of road objects is made possible thanks to a spectroradiometer. Reflectance spectroscopy in the visible and NIR range has been used for years as a nondestructive tool for evaluating soil properties and vegetation studies [5], [6]. Using the same physical concept, an original application of reflectance measurement technics is implemented in the Cerema Adverse Weather platform [7], [8]. This paper will focus on the measurement of spectral reflection of different road objects in the range from 350 to $2450 \mathrm{~nm}$. The choice of road targets is made according to the critical use cases studied for the development of autonomous vehicles and for their safety. In this first investigation, the measurements concern signaling equipment, fixed obstacles, or vulnerable road users, as pedestrians, who must be detected at a long distance, to guaranty the safety of autonomous driving.

\section{STUDY OF CRITICAL USE CASES AT CEREMA ADVERSE WEATHER PLATFORM}

\section{A. Critical use cases}

In order to evaluate the performance of autonomous vehicles, various use cases must be considered. The identification of relevant objects in the visual scene such as lane marking, or road signs is a key point for guidance and control of trajectory of the vehicle. The detection of vulnerable road users or critical objects is essential to avoid accidents and therefore demonstrate the safety of the system and allow traffic clearance on open lanes. Indeed, perception sensors as Lidars, are the entry points for this crucial information, that is transmitted to the vehicle control system. This control system will then decide of the action: e.g. emergency braking and/or avoidance manoeuvre. Thus, use cases may include various objects as: 
- in urban areas, vulnerable road users, pedestrians, cyclists and cars

- on motorways, lanes marking, road signs ...

- on any roads, fallen objects on the road as tires, exhaust pipes...

In facts, in a natural driving situation, environmental conditions are random, and it is not possible to repeat on request the same use cases. Therefore, testing facilities are needed to repeat the same scenarios multiple times, providing guarantees for metrological control when testing sensors. Examples of use cases investigated in the Cerema Adverse weather platform are displayed in Fig.1, for pedestrian crossing with vulnerable road users and for work zone with signaling equipments.
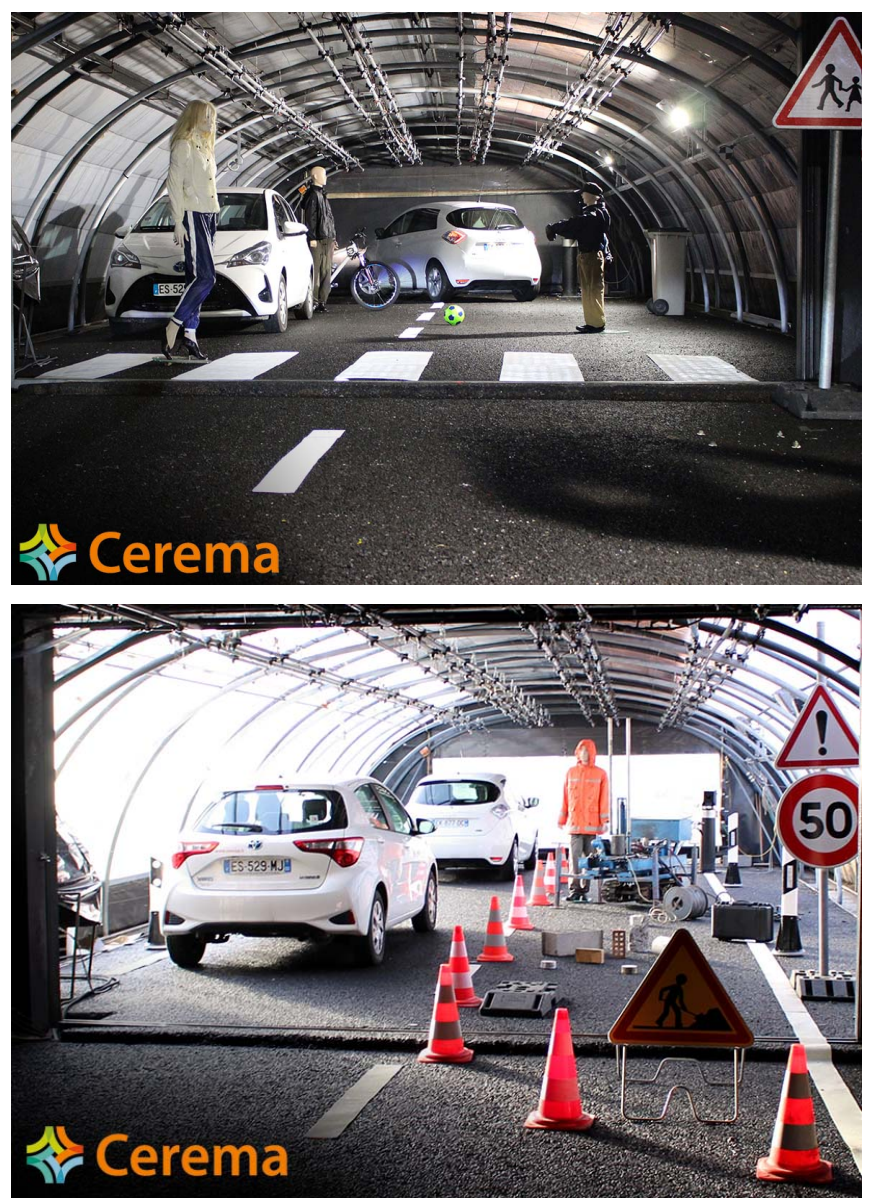

Fig. 1. Examples of use cases studied in the Cerema Adverse Weather Platform.

\section{B. The Cerema Adverse Weather Platform}

The Cerema Adverse Weather Platform, was developed to investigate all transports systems that could be affected by adverse conditions, including fog and rain [7], [8]. It allows to reproduce various scenarios, as detection of vulnerable roar users or fixed obstacles, in clear conditions, night conditions, and with various ranges of fog and rain precipitations on a total length of 30 meters (Fig.2).

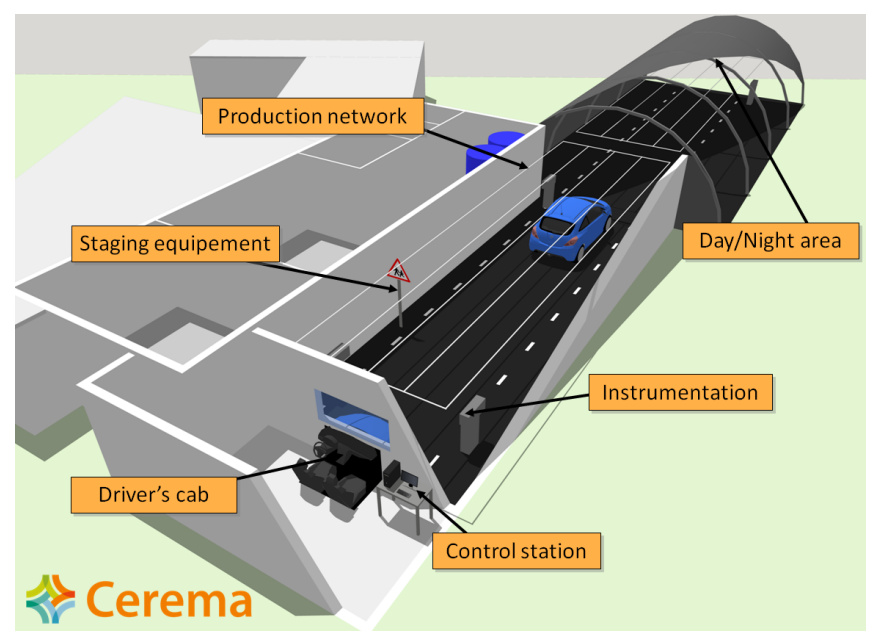

Fig. 2. The Cerema Adverse Weather platform [9].

Dedicated to research and development, It is also open to private companies looking for a testing facility with controlled conditions. It has been used for years in partnership or collaborative projects in order to investigate various scientific topics, as humans perception in adverse conditions [10], [11], vision systems capabilities in fog or rain conditions [12], [13], [14] or computer vision algorithms for objects detection [15], [4]. The physical characteristics of rain and fog produced in the platform are described in the recent study on Lidars performances in fog and rain [3].

This platform has got a high-level instrumentation to evaluate the performance of perceptual sensors for autonomous vehicles in adverse conditions. Some weather instruments are dedicated to characterizing the atmosphere in fog or rain conditions:

- transmissiometer, for meteorological visibility in fog from 5 to $1000 \mathrm{~m}$,

- optical granulometer, for fog droplet size distribution from 0.4 to $40 \mu \mathrm{m}$,

- rain gauge and a spectro-pluviometer for rainfall rate from 0.001 to $1200 \mathrm{~mm} / \mathrm{h}$.

In order to characterize the road environment itself and the object properties to be perceived by humans or by artificial vision sensors as cameras or Lidars, other devices are available:

- a video-photocolorimeter for Luminance from 0.003 to $50000 \mathrm{~cd} / \mathrm{m}^{2}$ in the visible range,

- reference cameras in visible, NIR, SWIR and LWIR,

- and a spectroradiometer for spectral reflectance of surfaces in the range of 350 to $2450 \mathrm{~nm}$.

\section{SPECTRAL REFLECTANCE MEASUREMENT}

\section{A. The PSR+3500 Spectroradiometer}

The spectroradiometer used in the Cerema Adverse weather platform is the PSR+3500 from Spectral Evolution (Fig.3). This type of device measures the radiance [16]. This radiometric quantity is expressed in $W / \mathrm{m}^{2} / \mathrm{nm} / \mathrm{sr}$. The measuring range is between $350 \mathrm{~nm}$ and $2450 \mathrm{~nm}$. 


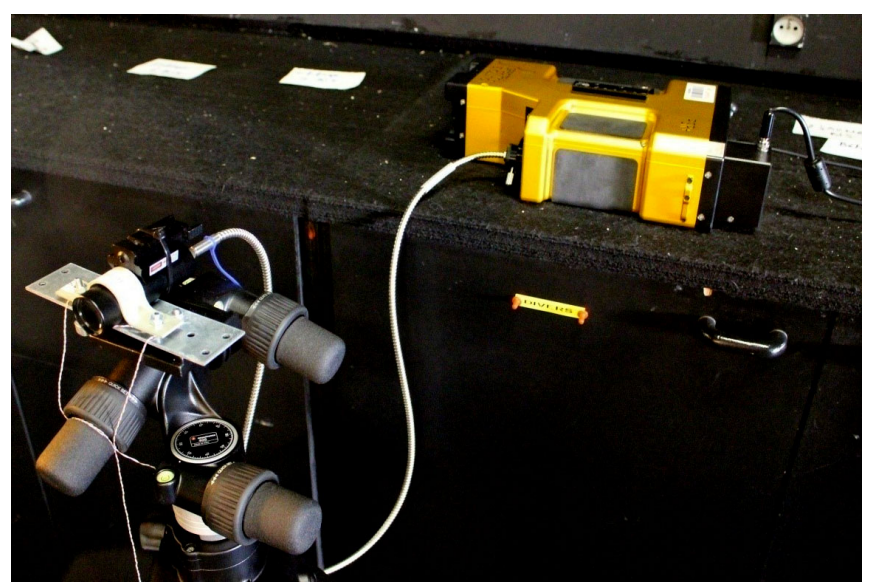

Fig. 3. The Spectral Evolution PSR +3500 spectroradiometer and its $1{ }^{\circ}$ aperture optics.

Light energy enters the spectroradiometer through the input optics. The light entering the sensor is collimated by a mirror. Each part of the spectrum must be directed to the photodiodes detectors corresponding to its spectral range. The sensor is composed of three photodiode arrays:

- A silicium detector measures from $350 \mathrm{~nm}$ to $1000 \mathrm{~nm}$ with a resolution of $3 \mathrm{~nm}$, and uncertainty of 1 to $4 \%$.

- A cooled InGaAS sensor operates in the spectral range $1000 \mathrm{~nm}$ to $1900 \mathrm{~nm}$ with a resolution of $8 \mathrm{~nm}$, and uncertainty smaller than $1 \%$.

- A second cooled InGaAS sensor measures the light power of radiation between $1900 \mathrm{~nm}$ and $2500 \mathrm{~nm}$ with a resolution of $6 \mathrm{~nm}$, and uncertainty of about $1 \%$.

\section{B. Experimental protocol}

The experimental protocol consists in carrying out a relative measurement of the reflectance of the target compared to a calibrated white target of uniform reflectance. In order to measure the spectral reflectance of object it is necessary to illuminate with a calibrated light source emitting in the corresponding wavelength range, a $400 \mathrm{~W}$ halogen lamp is used. During these tests, this lamp is at a fixed position, and the spectroradiometer changes of position, see Fig.4. The target is placed at a fixed distance $d$, at 2 meters from the halogen source. The beam emitted by the source is orthogonal to the target surface. The spectroradiometer, target and halogen lamp are in the same horizontal plan, at 1.20 meters above the ground. Measurements are made in a dark room. The spectroradiometer is first placed to obtain a measuring angle $\beta$ of $10^{\circ} . \beta$ is the angle formed between the source and the spectroradiometer in the horizontal plane. A reference measurement is made with a white calibrated target. Then the object to be characterized is installed in place of the calibrated target. To get the angular reflectance, the spectroradiometer is placed at angle $\beta$ from $10^{\circ}$ to $80^{\circ}$ in $10^{\circ}$ steps. The same protocol is repeated for the different targets.

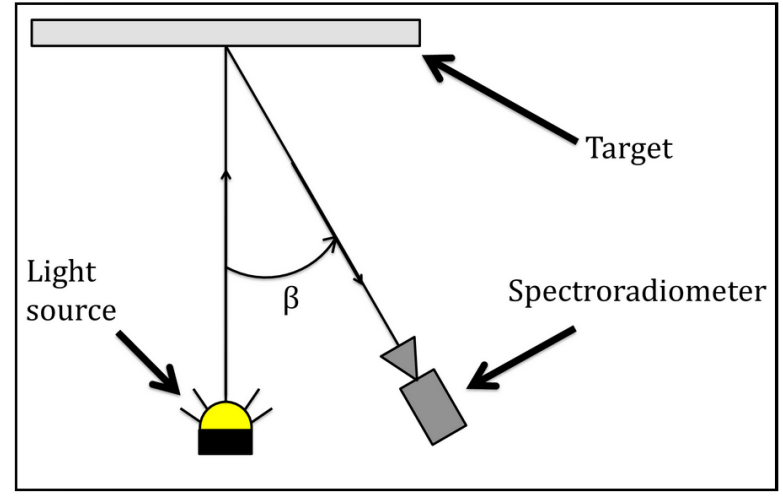

Fig. 4. Diagram of the experimental set-up, source, calibrated target, spectroradiometer.

\section{Reflectance calculation}

The spectral response represents the reflectance $R$ as a function of the wavelength $\lambda$. The reflectance $R$ of an object corresponds to the ratio of the reflected energy $r$ to the incident energy $E$ received by the object under study [16]:

$$
R=\frac{r}{E}
$$

In the case of the measurement set up, the reflected energy $r$ corresponds to the measurement of the radiance performed on the object at the different measurement angles. In order to calculate the incident energy, $E$, it is necessary, to measure a reference radiance $R a d_{r e f}$, on a calibrated white target. The incident energy $E$ calculated as follows using the spectral response of this reference target $R_{\text {ref }}$ :

$$
E=\frac{R a d_{r e f}}{R_{r e f}}
$$

Thus, by combining the two equations, it is possible to obtain the spectral response of the studied object:

$$
R=\frac{r}{R a d_{r e f}} R_{r e f}
$$

In order to evaluate the response of the targets as a function of the measurement angle, this formula is applied independently for each measurement angle. Three reference targets have been chosen for having a uniform reflection on the range of the studied wavelength: 350 to $2450 \mathrm{~nm}$. The reference calibrated targets are Zenith Polymer from Pro-Lite Technology (Fig.5).

\section{RESULTS}

\section{A. Validation of the spectral response of calibrated targets}

In order to validate the experimental protocol and reflectance calculation, we have measured the reflectance of two other calibrated targets having uniform reflectance. The spectral response measured is compared to the reference value given in the calibration certificate. Fig.6.a and 7.a show the results of the measurements of $R$, the spectral reflectance, respectively for the grey calibrated target $(50 \%$ reflectance) and the black calibrated target (5\% reflectance). 


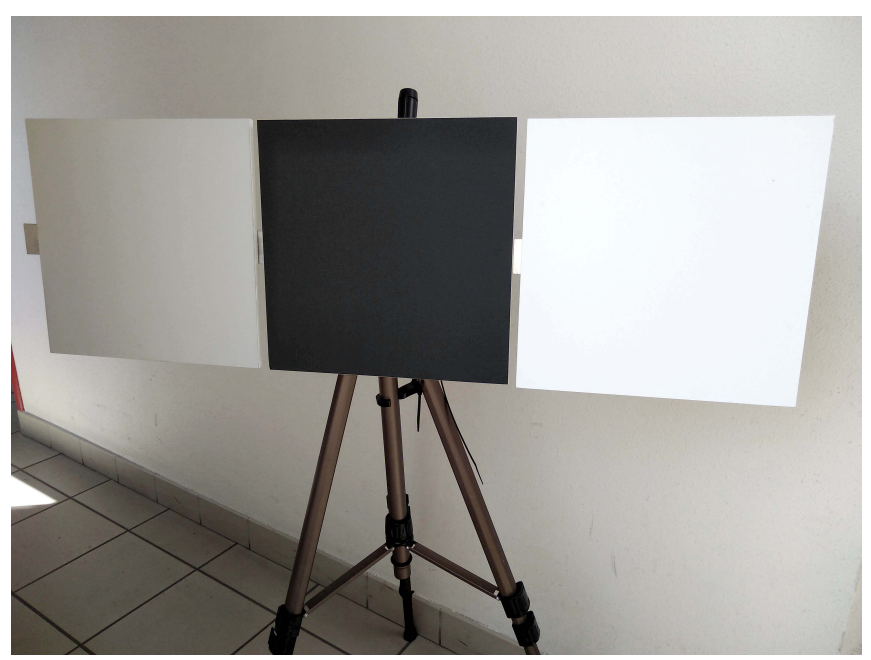

Fig. 5. The reference targets grey (50\%), black (5\%) and white (90\%) Zenith Polymer from Pro-Lite Technology.

On both figures, the measured values (red curve) is compared to the theoretical value (blue curve). It shows the agreement between measured and theoretical mean value and the uniformity of reflection with the wavelength. The uncertainty of the measured values is less than $5 \%$ for most part of the explored range. It should be noted that the measurement uncertainty increases beyond $2000 \mathrm{~nm}$. Fig.6.b and 7.b show the reflectance curves as a function of the angle between $10^{\circ}$ and $80^{\circ}$, for several wavelengths. Calibrated targets show Lambertian reflection. In the case of the black target, fig. 8.b, the radiation $\lambda=2400 \mathrm{~nm}$ doesnt seem to behave like the others. The comparison of the measured spectral reflectance on calibrated targets to the reference values given by the calibration certificates, shows a good agreement in the data for the mean value of reflectance and the uncertainty keep low values less than $5 \%$ on most part of the analyzed range. Therefore this validates the proposed methodology.

\section{B. Results of the measurements on road objects}

Based on the use cases described before, many measurements were made on a wide variety of road objects. We will present here a selection of objects according to their reflection characteristics: retroreflective targets, almost Lambertian objects and other objects categories. The selected retroreflective objects are:

- work zone signaling cone, with grey reflective strip;

- road reflector.

The selected quasi-Lambertian reflective objects are:

- asphalt pavement;

- for pedestrian clothing : dark blue polar sweater.

The other selected object on road surface are:

- tire;

- exhaust pipe.

In order to avoid increasing the complexity of the following figures, the uncertainty is not reported, it is of the same magnitude as above.

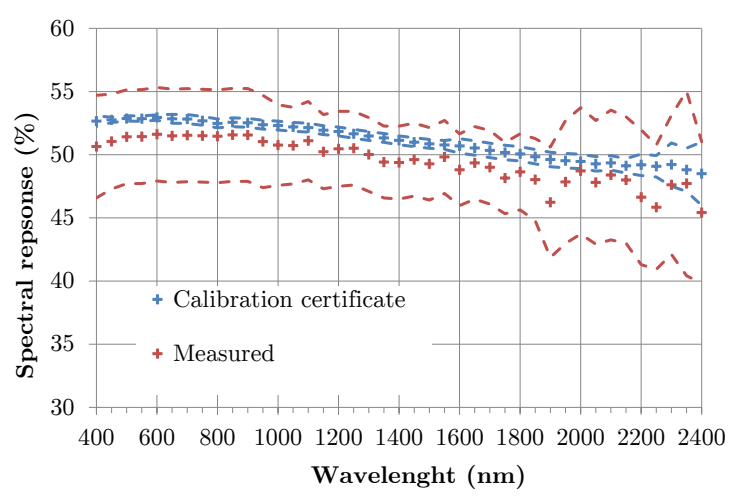

a. Spectral response at $10^{\circ}$.

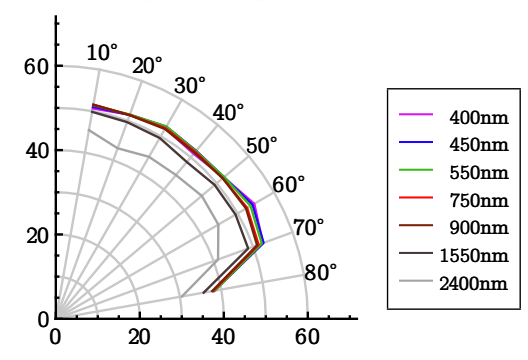

b. Angular distribution between $10^{\circ}$ and $80^{\circ}$.

Fig. 6. Spectral response of the grey target (50\% reflectance), with uncertainty.

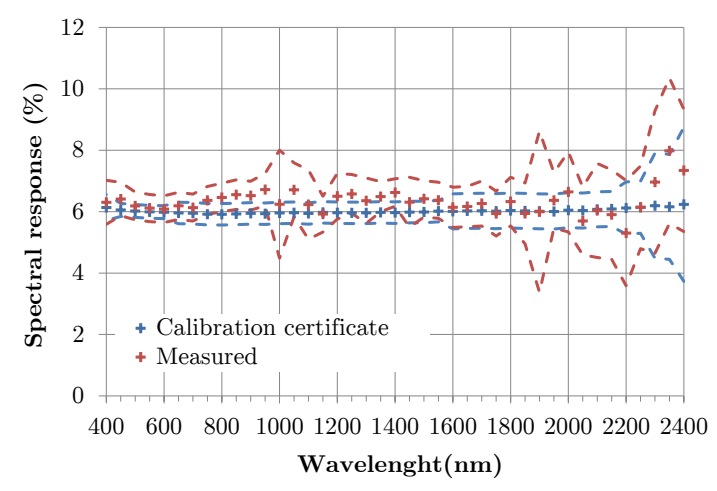

a. Spectral response at $10^{\circ}$

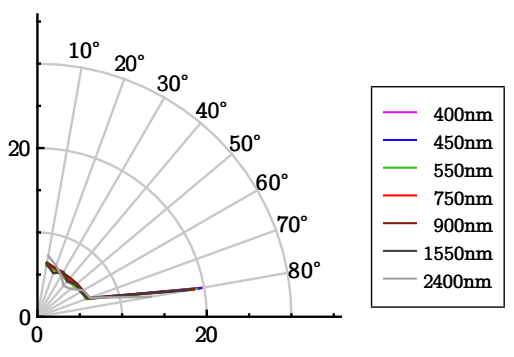

b. Angular distribution between $10^{\circ}$ and $80^{\circ}$.

Fig. 7. Spectral response of the black target (5\% reflectance), with uncertainty. 
Picture.

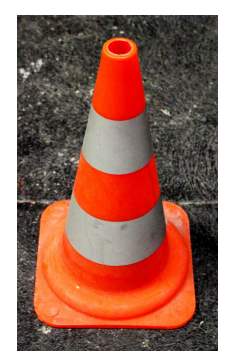

Wavelenght $(\mathrm{nm})$

Spectral response at $10^{\circ}$.

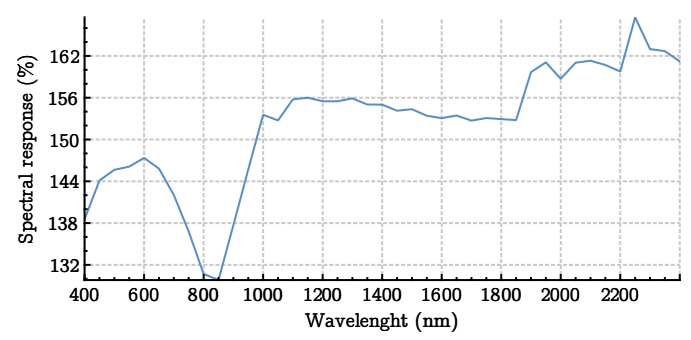

Angular distribution.

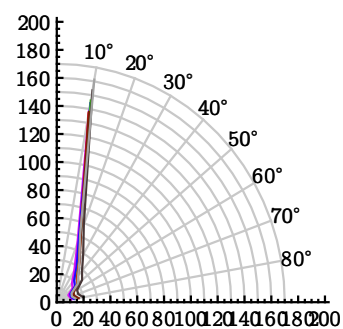

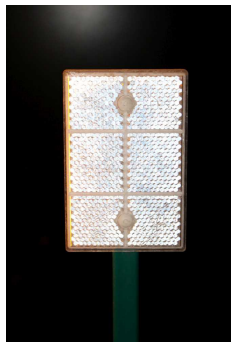
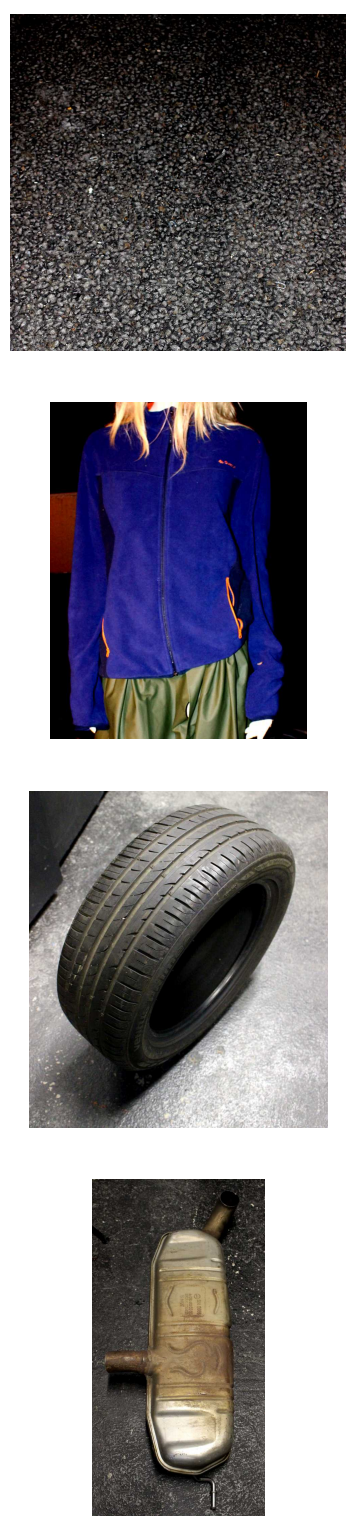
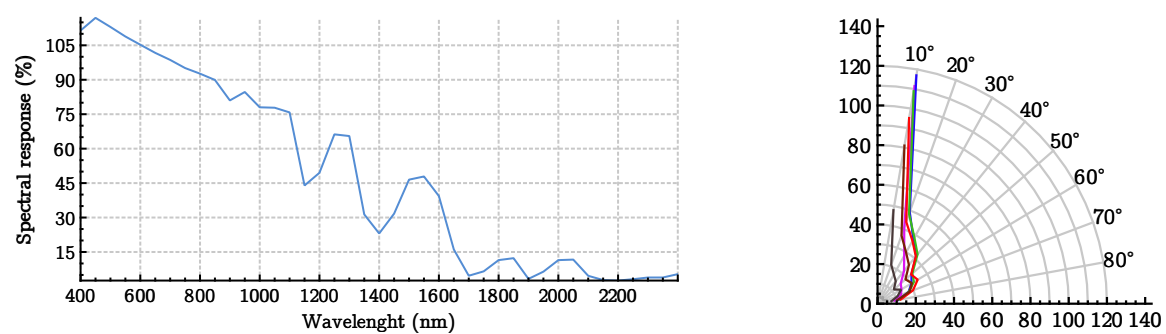

Fig. 9. The road reflector, spectral response and angular distribution.
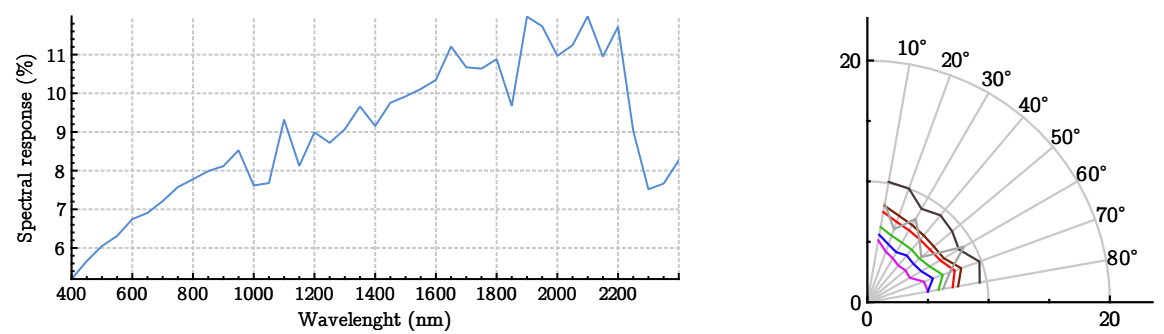

Fig. 10. Asphalt pavement, spectral response and angular distribution.
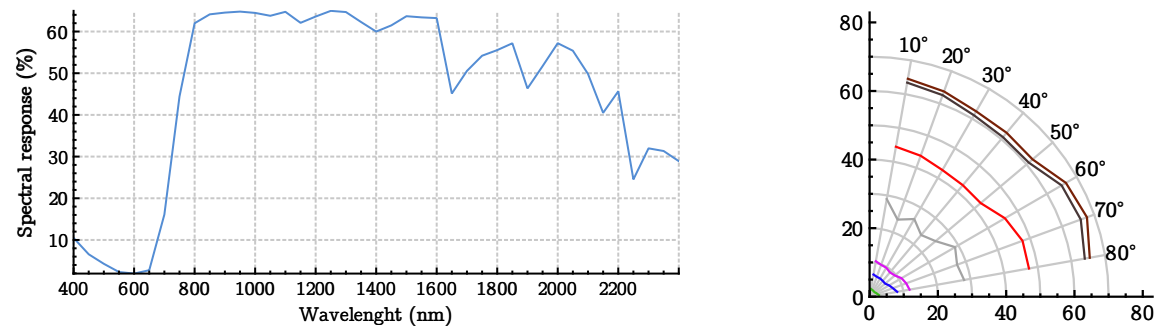

Fig. 11. The dark blue polar sweater, spectral response and angular distribution.
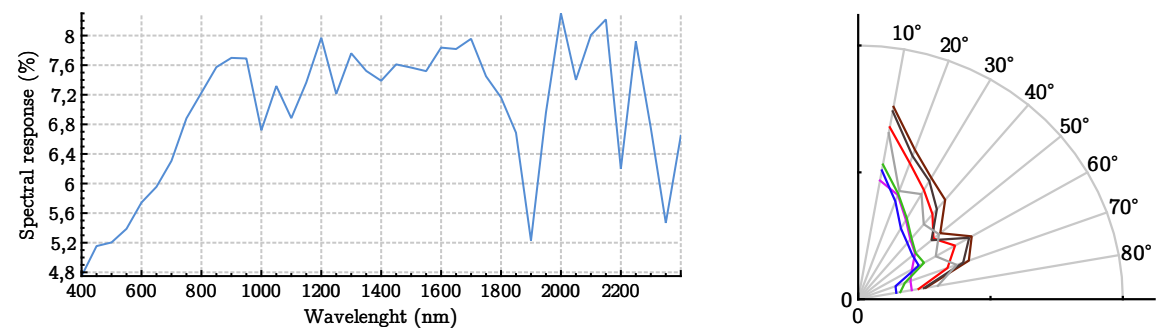

Fig. 12. The tire, spectral response and angular distribution.
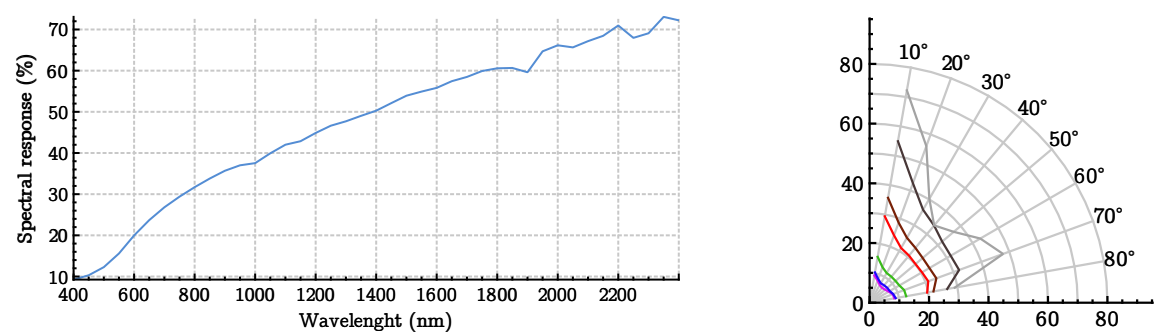

Fig. 13. The exhaust pipe, spectral response and angular distribution 
Figures 8 to 13 show from the left to the right, the object, its spectral response and the angular distribution of the reflectance. The analysis of each object reflectance gives the following results.

In Fig.8, the grey part of the cone is highly reflective for an angle of $10^{\circ}$. The reflectance does not change with the wavelength. Fig.9 shows that the reflector reflectance is decreasing with wavelength. The most reflected radiation is that of the visible. Beyond $1550 \mathrm{~nm}$, it reflects only $50 \%$ of the radiation, while its spectral response is negligible at 2400 $\mathrm{nm}$. It is therefore necessary to pay attention to this type of equipment, especially with Lidars using a $1550 \mathrm{~nm}$ laser. In Fig.10, the asphalt is not very reflective and it has a "quasiLambertian" behavior. Its spectral response does not exceed $10 \%$ in the infrared, with a maximum of $7.5 \%$ in the visible range. For the dark blue polar sweater, infrared wavelengths between $800 \mathrm{~nm}$ and $1550 \mathrm{~nm}$ are the more reflecting (Fig.11). The tire in Fig.12 has a higher reflectance between 750 and $1800 \mathrm{~nm}$ and about 2000 and $2400 \mathrm{~nm}$. A narrow band of lower reflectance is observed at about $1900 \mathrm{~nm}$. In Fig.13, spectral response of the exhaust pipe is increasing with the wavelengths. Indeed, the spectral response is low, $10 \%$ for the wavelength $400 \mathrm{~nm}$ while the spectral response for the same angle is $70 \%$ for the wavelength $2400 \mathrm{~nm}$. Its shiny appearance confirms the reflectance peak at $10^{\circ}$.

\section{Discussion}

As a general result, this inventory of the reflective properties of various road objects is very useful to consider, when interpreting camera or Lidars measurements dedicated to object detection. It can also be used for simulations. Some objects may be "visible", in the sense of detectable, with a high reflectance in some wavelengths, and maybe "not very perceptible", with low reflectance, in other wavelengths, e.g. the reflector on Fig.9. These results are in favor of the development of a sensors suite with various wavelength. Then data fusion of all measurements will optimize the system and even produce redundancy for the security of the system. These results of measurements show a zone of most important reflectance between 750 and $1550 \mathrm{~nm}$ that is much in favor for sensors development.

\section{CONCLUSION}

In the context of the development of the optical sensors for autonomous driving, the definition of a reference test to evaluate the performance of the sensors is necessary. As these sensors, as cameras or Lidars, cover wavelength ranges beyond the visible, the characterization of reflection properties of objects for all wavelengths ranges, is a prerequisite for a good understanding of the factors influencing the performance i.e. the ability to detect objects. This study highlights the need to characterize the spectral reflectance of road objects at all wavelengths. This first characterization in a "clear" atmosphere could be combined with the atmospheric attenuation effect in the case of adverse situations such as rain or fog, for a complete overview of the most critical situations for driving autonomous vehicles. These original data could also contribute to computer vision modelling and simulation of sensors detection for various objects.

\section{ACKNOWLEDGMENT}

The authors would like to thank Jean-Luc Bicard and Anthony Bayle for their active contribution to experiments and data processing.

\section{REFERENCES}

[1] SAE International, "J3016 - Taxonomy and Definitions for Terms Related to On-Road Motor Vehicle Automated Driving Systems," p. $12,2014$.

[2] S. Shladover, "Technical Challenges for Fully Automated Driving Systems," in ITS World Congress, Detroit, 2014.

[3] M. Kutila, P. Pyykönen, H. Holzhüter, M. Colomb, and P. Duthon, "Automotive LiDAR performance verification in fog and rain," in 21 st International Conference on Intelligent Transportation Systems, 2018.

[4] M. Bijelic, F. Mannan, T. Gruber, W. Ritter, K. Dietmayer, and F. Heide, "Seeing Through Fog Without Seeing Fog: Deep Sensor Fusion in the Absence of Labeled Training Data," CoRR, vol. abs/1902.0, 2019. [Online]. Available: http://arxiv.org/abs/1902.08913

[5] R. Viscarra Rossel, D. J. J. Walvoort, A. Mcbratney, L. Janik, and J. O. Skjemstad, "Visible, Near Infrared, Mid Infrared or Combined Diffuse Reflectance Spectroscopy for Simultaneous Assessment of Various Soil Properties," Geoderma, vol. 131, pp. 59-75, 2006.

[6] A. Ghulam, J. Fishman, M. Maimaitiyiming, J. L. Wilkins, M. Maimaitijiang, J. Welsh, B. Bira, and M. Grzovic, "Characterizing Crop Responses to Background Ozone in Open-Air Agricultural Field by Using Reflectance Spectroscopy," IEEE Geoscience and Remote Sensing Letters, vol. 12, no. 6, pp. 1307-1311, 2015.

[7] M. Colomb, K. Hirech, P. André, J. Boreux, P. Lacôte, and J. Dufour, "An innovative artificial fog production device improved in the European project FOG," Atmospheric Research, vol. 87, no. 3-4, pp. 242-251, mar 2008.

[8] P. Duthon, F. Bernardin, F. Chausse, and M. Colomb, "Methodology used to evaluate computer vision algorithms in adverse weather conditions." in Proceedings of 6th Transport Research Arena, Warsaw, Poland, 2016.

[9] "Cerema Adverse Weather Platform," 2018. [Online]. Available: https://www.cerema.fr/fr/innovation-recherche/innovation/offrestechnologie/plateforme-simulation-conditions-climatiques-degradees

[10] V. Cavallo, M. Colomb, and J. Doré, "Distance Perception of Vehicle Rear Lights in Fog," Human Factors, vol. 43, no. 3, pp. 442-451, 2001. [Online]. Available: https://doi.org/10.1518/001872001775898197

[11] B. Quétard, J.-C. Quinton, M. Colomb, G. Pezzulo, L. Barca, M. Izaute, O. K. Appadoo, and M. Mermillod, "Combined effects of expectations and visual uncertainty upon detection and identification of a target in the fog," Cognitive processing, vol. 16, no. 1, pp. 343348, 2015.

[12] F. Bernardin, R. Bremond, V. Ledoux, M. Pinto, S. Lemonnier, V. Cavallo, and M. Colomb, "Measuring the effect of the rainfall on the windshield in terms of visual performance," Accident Analysis and Prevention, vol. 63, pp. 83-88, 2014.

[13] M. Marchetti, V. Boucher, J. Dumoulin, and M. Colomb, "Retrieving visibility distance in fog combining infrared thermography, Principal Components Analysis and Partial Least-Square regression," Infrared Physics and Technology, vol. 71, pp. 289-297, 2015. [Online]. Available: http://dx.doi.org/10.1016/j.infrared.2015.05.002

[14] N. Pinchon, O. Cassignol, A. Nicolas, P. Leduc, J. P. Tarel, R. Bremond, G. Julien, N. Pinchon, O. Cassignol, A. Nicolas, F. Bernardin, N. Pinchon, O. Cassignol, A. Nicolas, F. Bernardin, P. Leduc, and J.-p. Tarel, "All-weather vision for automotive safety : which spectral band ?" in International Forum on Advanced Microsystems for Automotive Applications, 2016.

[15] K. Dahmane, P. Duthon, F. Bernardin, M. Colomb, N. Essoukri Ben Amara, and F. Chausse, "The Cerema pedestrian database : A specific database in adverse weather conditions to evaluate computer vision pedestrian detectors," in 7th Conference on Sciences of Electronics, Technologies of Information and Telecommunications (SETIT), 2016, pp. 480-485.

[16] J. R. Howell, M. P. Menguc, and R. Siegel, Thermal radiation heat transfer. CRC press, 2015. 